\title{
Harnessing the Power of Radionuclides for Optical Imaging: Cerenkov Luminescence Imaging
}

\author{
Yingding Xu, Hongguang Liu, and Zhen Cheng \\ Molecular Imaging Program at Stanford (MIPS), Department of Radiology and Bio-X Program, Stanford University, \\ Stanford, California
}

\begin{abstract}
Over the past several years, nuclear imaging modalities such as PET and SPECT have received much attention because they have been instrumental not only in preclinical cancer research but also in nuclear medicine. Yet nuclear imaging is limited by high instrumentation cost and subsequently low availability to basic researchers. Cerenkov radiation, a relativistic physical phenomenon that was discovered 70 years ago, has recently become an intriguing subject of study in molecular imaging because of its potential in augmenting nuclear imaging, particularly in preclinical small-animal studies. The intrinsic capability of radionuclides emitting luminescent light from decay is promising because of the possibility of bridging nuclear imaging with optical imaging - a modality that is much less expensive, is easier to use, and has higher throughput than its nuclear counterpart. Thus, with the maturation of this novel imaging technology using Cerenkov radiation, which is termed Cerenkov luminescence imaging, it is foreseeable that advances in both nuclear imaging and preclinical research involving radioisotopes will be significantly accelerated in the near future.
\end{abstract}

Key Words: Cerenkov luminescence imaging; radionuclides; optical imaging; PET

J Nucl Med 2011; 52:2009-2018

DOI: 10.2967/jnumed.111.092965

\section{$\mathbf{M}$} olecular imaging has emerged as one of the most promising fields in biomedical research in recent years $(1,2)$. The advantage of noninvasively imaging cellular processes both in vitro and in vivo promises great potentials in drug and biomarker development, early diagnosis of cancer, cardiovascular and neurologic diseases, and novel treatment methods targeting these pathologies.

Molecular imaging uses a diversified group of modalities including MRI, ultrasound, PET, SPECT, and optical imaging (OI). Each modality has its own advantages and disadvantages. For instance, nuclear imaging modalities such as PET have high sensitivity and excellent quantifi-

\footnotetext{
Received May 10, 2011; revision accepted Jul. 29, 2011.

For correspondence or reprints contact: Zhen Cheng, Molecular Imaging Program at Stanford, Department of Radiology and Bio-X Program, 1201 Welch Rd., Lucas Expansion, P095, Stanford University, Stanford, CA 94305. E-mail: zcheng@stanford.edu

Published online Nov. 11, 2011.

COPYRIGHT @ 2011 by the Society of Nuclear Medicine, Inc.
}

cation capability but suffer from poor spatial resolution, which is confined to millimeter range. On the other hand, MRI features submillimeter spatial resolution but is limited by low sensitivity and the high cost of instrumentation. Much less expensive and more widely available than PET and MRI, traditional OI also features high sensitivity, short scanning time, and relatively high throughput, yet its potential has been mostly constrained to preclinical studies because of low penetration and high scattering of optical signals in living tissues.

In the past 2 years, Cerenkov radiation (CR) has been a novel topic in molecular imaging and has been demonstrated by several groups to have significant potentials in bioimaging (3). Discovered back in 1934, CR is an electromagnetic radiation emitted when a charged particle such as a positron travels at a speed beyond the speed of light in a dielectric medium $(4,5)$. As the charged particle travels through the medium, it disrupts the electromagnetic field of the medium and temporarily displaces the electrons in the atoms of the medium. Photons are emitted when the displaced electrons return to the ground state after the disruption has ceased. A superluminal speed of the particle is required for $\mathrm{CR}$ to occur because the emitted photons would otherwise destructively interfere with each other and no radiation would result. A dielectric insulating medium is also essential because a conductor is not electrically polarizable and therefore would not respond to charged particles. CR is named after Russian physicist Pavel Alekseyevich Cerenkov, winner of the 1958 Nobel Prize in Physics, who first described the phenomenon. Ilya Frank and Igor Tamm, 2 Russian physicists who shared the Nobel Prize with Cerenkov, further advanced the theory of their colleague. Their contributions include the FrankTamm formula, which dictates that the relative intensity of $\mathrm{CR}$ at a certain frequency is approximately proportional to that frequency. Together with the fact that the sensitivity of the human eye peaks around green, this formula helps explain why CR always appears to be brilliant blue, most notably observed around nuclear reactors.

\section{PHYSICS OF CR}

High-energy charged particles such as positrons released by ${ }^{18} \mathrm{~F}$ behave according to physics of relativity because 
immediately after emission from the nucleus, the speed of these particles often reaches close to the speed of light in vacuum $(c)$. Incidentally in media such as biologic tissue and water, the speed of light $\left(v_{n}\right)$ is less than $c$ and is determined by the refractive index of the medium $(n)$ :

$$
v_{n}=\frac{c}{n} .
$$

The relativistic kinetic energy of a certain particle $(E)$ with its speed close to $c$ is determined by its mass $(m)$ and its speed $(v)$ :

$$
E=m c^{2}\left(\frac{1}{\sqrt{1-v^{2} / c^{2}}}-1\right)
$$

Positrons produced by ${ }^{18} \mathrm{~F}$, with the maximum relativistic kinetic energy of $635 \mathrm{keV}$, will have a calculated initial speed of $0.90 c$ using Equation 2-a speed that is significantly higher than the calculated speed of light of $0.75 c$ in water using Equation 1. According to the mechanism of CR, as long as these positrons have a superluminal speed in a dielectric medium $\mathrm{CR}$ will be produced until interactions with the medium cause these particles to lose kinetic energy to the point that their speed, $v$, drops below the speed of light in that medium, $v_{n}$. Thus, this threshold particle kinetic energy that is required for $\mathrm{CR}$ in a particular medium can also be derived by combining Equations 1 and 2, and in biologic tissue this threshold was calculated to be approximately $260 \mathrm{keV}$, which is significantly lower than $635 \mathrm{keV}$, the maximum kinetic energy of ${ }^{18} \mathrm{~F}$ positrons $(6,7)$.

Ilya Frank and Igor Tamm first calculated the number of photons produced by $\mathrm{CR}$, and later Ross published a table of numerically integrated solutions using the Frank-Tamm equations (8). Robertson et al. recently used this table to estimate that a $635-\mathrm{keV}$ positron would produce approximately 20 photons, with wavelengths ranging from 250 to $600 \mathrm{~nm}$, and one ${ }^{18} \mathrm{~F}$ decay would produce about 3 photons in average in water (9). Spinelli et al. also presented an analytic model of CR spectrum of the positrons (10); according to their derived expression, whereas the magnitude of CR depends on energy spectrum of the particular positron, the intensity exhibits a dependence on the inverse of wavelength squared, $1 / \lambda^{2}$.

\section{RECENT ADVANCES IN CERENKOV LUMINESCENCE IMAGING (CLI)}

In 2009, Robertson et al. published a proof-of-principle work that applied CR to the field of molecular imaging (9). As a preliminary demonstration, they successfully detected luminescent signals from a gradient of concentrations of 2 high-energy positron-emitting radionuclides, ${ }^{18} \mathrm{~F}$ and ${ }^{13} \mathrm{~N}$ (both nuclides have maximum $\beta^{+}$energy exceeding the Cerenkov threshold), using Xenogen IVIS devices without fluorescence filters or an external excitation source (the result of ${ }^{18} \mathrm{~F}$ is shown in Fig. 1A).
Moreover, the measured charge-coupled device signal showed a positive correlation with refractive index of the medium, and light output also corresponded to the inverse of wavelength squared, both of which are consistent with $\mathrm{CR}$ (the latter result is shown in Fig. 1B). Using ${ }^{18} \mathrm{~F}$, Robertson et al. (9) also showed that half-life of signal intensity corresponded well to the half-life of the radioisotope. The authors then obtained ${ }^{18}$ F-FDG PET scans in tumor-bearing mice and quasiquantitatively showed a good correlation between signals from PET and radioactive OI. They coined the term CLI for this novel molecular imaging method in which visible light emission from $\mathrm{CR}$ is detected during decay of positron-emitting radionuclides.

With a different perspective regarding the applications of $\mathrm{CR}$ in the biomedical field, Cho et al., from the University of California Los Angeles, reported their work of using CR as a method to quantify $\beta$-particles in a microfluidic chip (11). The details of this work are discussed in the "Applications in Biomedical Research" section. It suffices to say that Cho et al. devised this novel system using CR from the decay of $\beta$-particles to quantitatively image microfluidic applications while achieving satisfactory spatial resolution, minimum detectable activity, and dynamic range (selected results are shown in Figs. 1C and 1D).

Around the same time, Spinelli et al. presented a detailed derivation of the CR spectrum (the "Physics of CR" section) as well as 2 methods of in vivo source-depth measurement, which will be discussed in greater detail in the "Applications in Biomedical Research" section (10). The authors also performed ${ }^{18} \mathrm{~F}-\mathrm{FDG}$ in vivo dynamic imaging using radioactive $\mathrm{OI}$ and demonstrated that the kinetics of ${ }^{18} \mathrm{~F}-\mathrm{FDG}$ uptake in mice can be studied with CLI.

Meanwhile, our group was independently and systematically studying the applicability of using radionuclides for OI, and a variety of radionuclides including $\beta^{+}-, \beta^{-}$, and $\gamma$-emitters were evaluated (12). As is known in nuclear physics, radionuclides decay via a variety of modes including $\alpha, \beta^{+}, \beta^{-}$, electron capture and isomeric transition. $\beta^{+}$-emitters include ${ }^{11} \mathrm{C},{ }^{13} \mathrm{~N},{ }^{15} \mathrm{O},{ }^{18} \mathrm{~F},{ }^{64} \mathrm{Cu},{ }^{68} \mathrm{Ga}$, and ${ }^{124} \mathrm{I}$; Robertson et al. used ${ }^{13} \mathrm{~N}$ and ${ }^{18} \mathrm{~F}$ to demonstrate CLI (9). On the other hand, ${ }^{3} \mathrm{H},{ }^{14} \mathrm{C},{ }^{32} \mathrm{P},{ }^{89} \mathrm{Sr},{ }^{90} \mathrm{Y},{ }^{131} \mathrm{I}$, and ${ }^{177} \mathrm{Lu}$ emit principally high-energy $\beta^{-}$, and the decay of these emitters is often accompanied by $\gamma$-rays. Importantly, many $\beta^{-}$-emitters have already been used for treatment of cancer. Electron capture occurs when an extranuclear electron is captured by the nucleus; ${ }^{67} \mathrm{Ga},{ }^{111} \mathrm{In},{ }^{123} \mathrm{I}$, and some other radioisotopes belong to this category. ${ }^{99 \mathrm{~m}} \mathrm{Tc}$, the most commonly used radionuclide in nuclear medicine, has the decay mode of isomeric transition whereby excited nuclei in the meta state emit $\gamma$-rays before returning to the ground state. We tested $2 \beta^{+}$-emitters $\left({ }^{18} \mathrm{~F}\right.$ and $\left.{ }^{64} \mathrm{Cu}\right), 3 \beta^{-}$-emitters $\left({ }^{90} \mathrm{Y}\right.$, ${ }^{131} \mathrm{I}$, and $\left.{ }^{177} \mathrm{Lu}\right), 1$ electron capture radionuclide $\left({ }^{111} \mathrm{In}\right)$, and the pure $\gamma$-emitter ${ }^{99 \mathrm{~m}} \mathrm{Tc}$ for optical signals, detection sensitivity, and spectrum (Fig. 2).

With the exception of ${ }^{99 \mathrm{~m}} \mathrm{Tc}$, all tested radionuclides showed good sensitivity, with 1- to 5-min acquisition times 


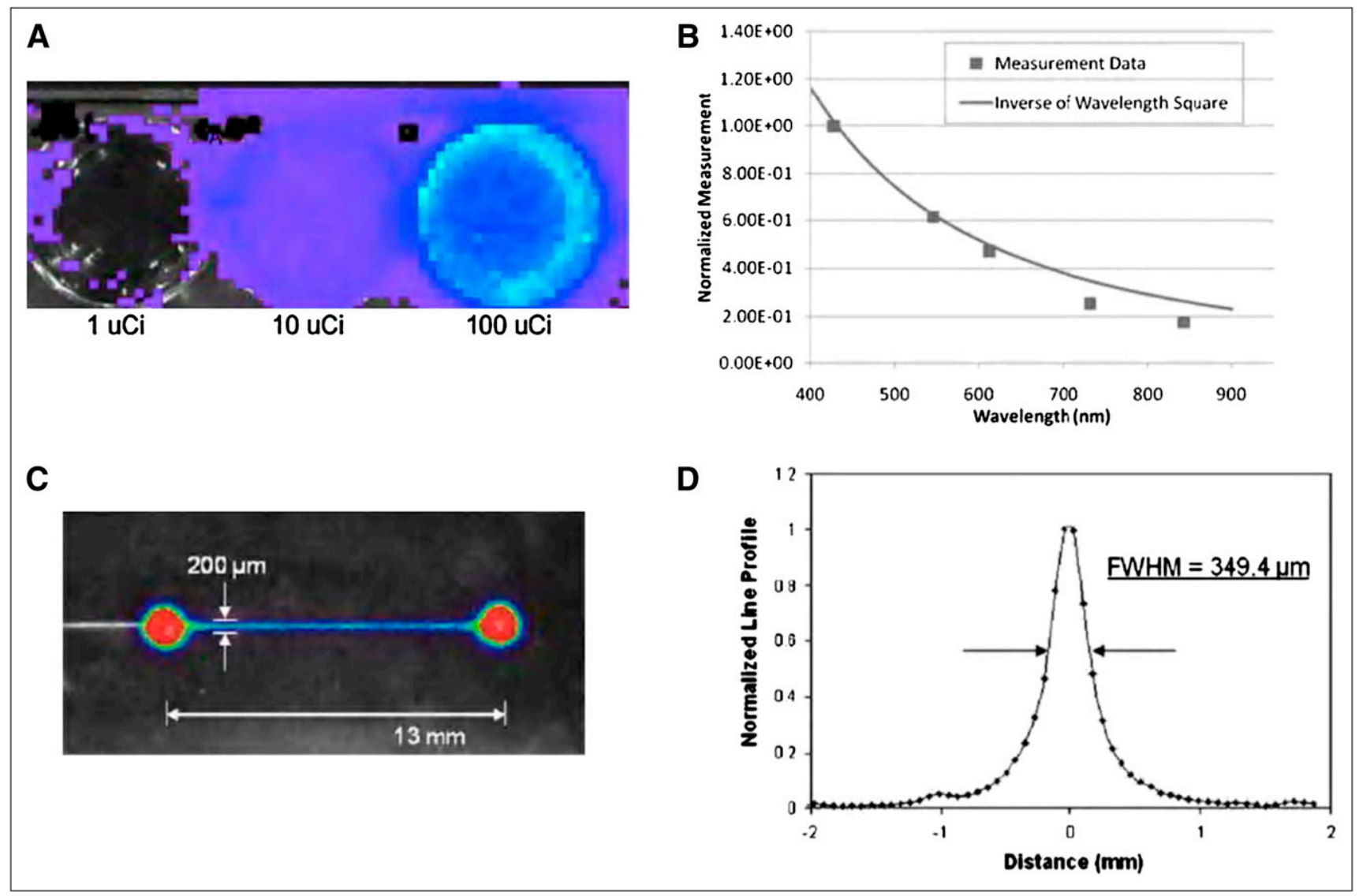

FIGURE 1. Proof-of-principle work for CLI. (A) Luminescence detected from well plates of 1,10 , and $100 \mu \mathrm{Ci}$ of $18 \mathrm{~F}$ after $10-\mathrm{s}$ image acquisition. (B) Results from series of filtered scans on wavelength of light detected from positron-emitter samples. Most of light is produced in blue portion of spectrum, which is expected for CR and follows inverse relationship with square of wavelength. Cerenkov light extends into green and red regions of electromagnetic spectrum, which could increase range of applications for in vivo imaging studies. (C) Artificially colored Cerenkov signal image overlaid on room light photograph of line channel with $200-\mu \mathrm{m}$ width. (D) Line profile of microchannel. This resolution was dominated by physical size of channel. ( $A$ and $B$ adapted with permission of (9) and $C$ and $D$ adapted with permission of (11).) $\mathrm{FWHM}=$ full width at half maximum.

(Fig. 2A). ${ }^{99 \mathrm{~m} T c}$ displayed almost zero intensity of light when observed by OI modalities, which is in agreement with a charged particle being one of the requirements of CR. Black and white paper were also used to cover the wells, and the observed shielding effect showed that detected luminescence was not due to $\beta$-emissions. By plotting average radiance $\left(\mathrm{p} / \mathrm{s} / \mathrm{cm}^{2} / \mathrm{sr}\right)$ versus activity $(\mathrm{Bq})$, we determined that the 3 most sensitive radionuclides were ${ }^{18} \mathrm{~F},{ }^{90} \mathrm{Y}$, and ${ }^{131}$ I (Fig. 2B). Figure 2C shows a roughly inverse relationship between the percentages of optical signal intensity and wavelengths, and this characteristic is preserved across all tested radionuclides except for ${ }^{99} \mathrm{~m} \mathrm{Tc}$. A phantom imaging study was then performed for ${ }^{18} \mathrm{~F},{ }^{90} \mathrm{Y}$, and ${ }^{131} \mathrm{I}$, and it was found that a high spatial resolution $(1.2 \mathrm{~mm})$ could be achieved by radioactive OI, similar to the findings of Cho et al. (11).

Our group then validated the in vivo applicability of radioactive OI with a triple-modality strategy using ${ }^{18} \mathrm{~F}-$ FDG in mice implanted with firefly luciferase (FLuc)transfected rat C6 glioma cells (C6-FLuc). The xenografts were imaged using bioluminescence imaging (BLI) (Supplemental Fig. 1A; supplemental materials are available online only at http://jnm.snmjournals.org), radioactive OI (Supplemental Fig. 1B), and PET (Supplemental Fig. 1C). The imaging results were further characterized by opening the thorax of the mice and identifying the tumor and heart and removing the organs and tissues. Additionally, signals from $\mathrm{Na}^{18} \mathrm{~F}$ bone accumulation in vivo were detected via both OI and PET, and quantification analyses for both modalities showed similar trends. The pure $\beta^{-}$-emitting SPECT probe $\mathrm{Na}^{131} \mathrm{I}$ was also tested, and good signals were observed in the thyroid gland as early as 30 min after injection (Supplemental Fig. 1D). Notably, our group labeled a heterodimeric peptide RGD-BBN (Arg-Gly-Asp coupled with bombesin for dual targeting of integrin $\alpha_{v} \beta_{3}$ and gastrin-releasing peptide receptor) with the clinically important pure $\beta^{-}$-emitter ${ }^{90} \mathrm{Y}$ and achieved good tumor-to-normal tissue (T/N) ratios (Supplemental Fig. 1E).

For ${ }^{18} \mathrm{~F}-\mathrm{FDG}$, time-lapse quantitative analysis of both radioactive OI and PET images of C6-FLuc mice was also performed (Supplemental Figs. 1F and 1G), and an excellent correlation of tumor uptake was found between measured 
FIGURE 2. Optical signals are detectable by $\mathrm{OI}$ instruments and have continuous

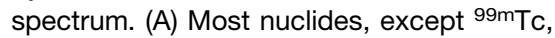
provide Ol signals with sensitivity within $0.004-$ to $0.370-\mathrm{MBq}(0.1-10 \mu \mathrm{Ci})$ range $\left({ }^{18} \mathrm{~F}: 5,2,1\right.$, and $0.1 \mu \mathrm{Ci} ;{ }^{131} \mathrm{I}: 10,5,1$, and $0.1 \mu \mathrm{Ci}$; ${ }^{90} \mathrm{Y}$ : $5,1,0.2$, and $0.01 \mu \mathrm{Ci}$; ${ }^{64} \mathrm{Cu}$ : 10, 5, 1, and $0.1 \mu \mathrm{Ci}$; ${ }^{177} \mathrm{Lu}: 10,5,2$, and 0.5 $\mu \mathrm{Ci} ;{ }^{111} \mathrm{In}: 10,5,1$, and $0.5 \mu \mathrm{Ci}$; and ${ }^{99 m \mathrm{TC}}$ : $20,10$, and $5 \mu \mathrm{Ci})$. (B) Detection sensitivity of different radionuclides. Radionuclides with higher slope value have stronger signal intensity. (C) Similar spectra were observed from different radionuclides. Results are presented as ratio of photons detected using narrow band emission filter (20-nm bandwidth) vs. total photons detected without filter. (Adapted with permission of (12).)

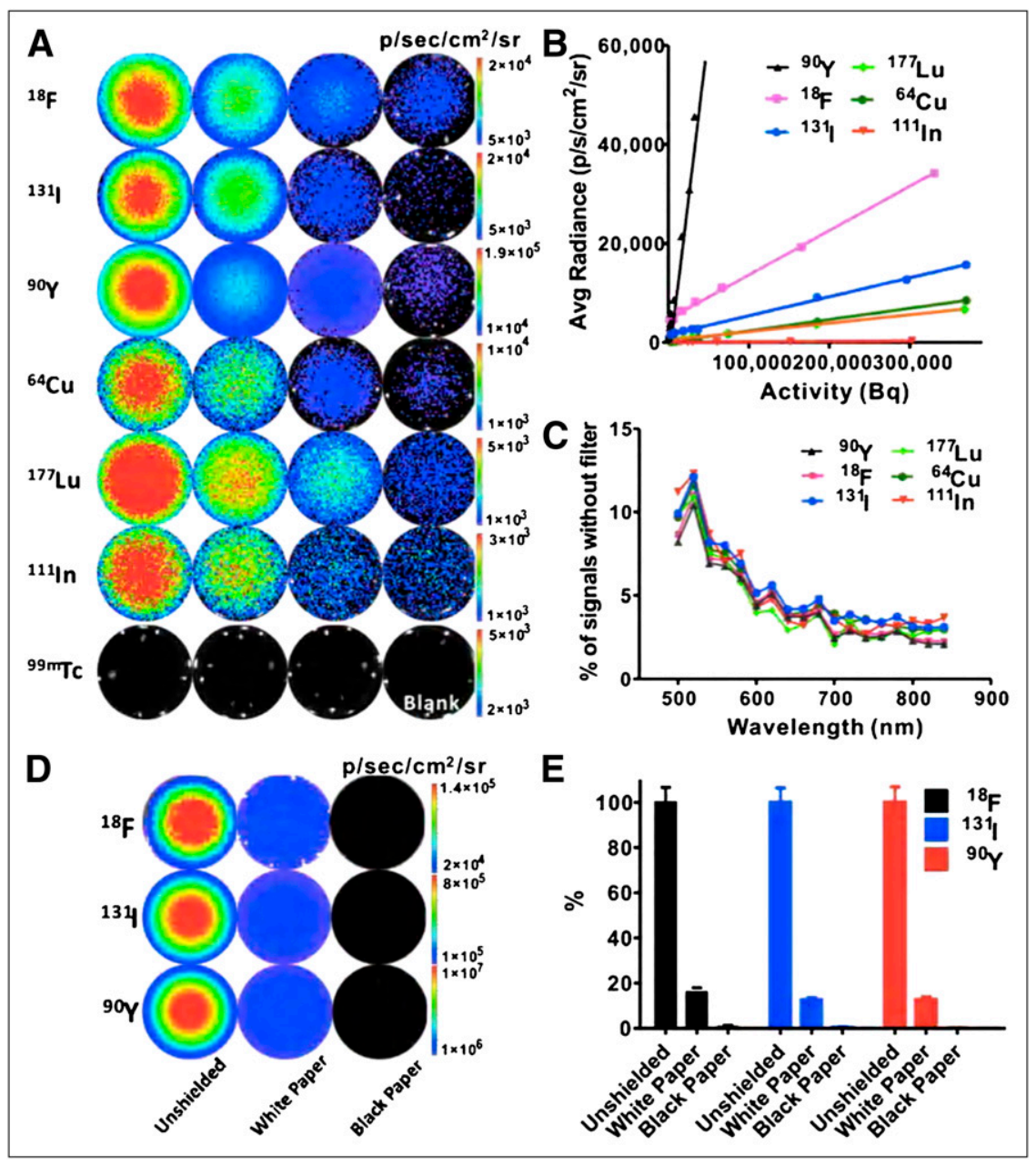

radiance from radioactive OI and percentage injected dose per gram from PET (Supplemental Fig. 1H, $R^{2}>0.95$ ).

\section{CROSS VALIDATIONS OF CLI}

\section{PET}

Thus far, PET has been the single most common method of cross validations of CLI, and most publications concerning CLI have presented PET data for comparison with OI. Therefore, only a few of these results will be discussed in this section. As previously mentioned, Robertson et al. looked at 2 mice, each bearing a flank colon tumor, and compared the standardized uptake value and percentage injected dose per gram from PET with light intensity from OI, finding that the results obtained from the 2 imaging techniques were consistent (9). Our group independently performed a systematic validation with both PET and BLI in vivo at multiple time points and found an excellent correlation between OI and PET signals $\left(R^{2}=0.97\right)(12)$. Recently, our group also demonstrated that the reporter gene-nuclear reporter probe system (herpes simplex virus type 1 thymidine kinase and $9-\left(4-{ }^{18} \mathrm{~F}-\right.$ fluoro-3-[hydroxymethyl] butyl) guanine $\left.\left[{ }^{18} \mathrm{~F}-\mathrm{FHBG}\right]\right)$ could be successfully imaged by OI both in vitro and in vivo (13). Statistical anal- ysis showed that both $\mathrm{OI}$ and PET displayed good T/N ratios. Examining a range of radionuclides including ${ }^{18} \mathrm{~F},{ }^{64} \mathrm{Cu},{ }^{89} \mathrm{Zr}$, ${ }^{124} \mathrm{I}$, and ${ }^{131} \mathrm{I}$ and the $\alpha$-emitter ${ }^{225} \mathrm{Ac}$, Ruggiero et al. found that radiance detected by OI and radioactivity detected by PET correlated well in a linear regression model $(R=0.98$ for ${ }^{89} \mathrm{Zr}$ ) (14). They also used a radiolabeled prostate-specific membrane antigen targeting the monoclonal antibody ${ }^{89} \mathrm{Zr}$ desferrioxamine B [DFO]-J591 for immuno-PET in LNCaP tumor-bearing mice positive for prostate-specific membrane antigen, and the data correlated well both qualitatively and quantitatively with CLI. As another example, Park et al. injected ${ }^{124}$ I-labeled trastuzumab (Herceptin; Genetech) into HER2-expressing NIH3T6.7 tumor-bearing mice and obtained OI and PET images, both of which showed good signals from tumors (15).

In summary, PET validations of CLI have been accomplished using many radiolabeled molecules as well as radionuclides alone, including some of the most widely used agents in nuclear medicine such as ${ }^{18} \mathrm{~F}-\mathrm{FDG}, \mathrm{Na}^{18} \mathrm{~F}$, and ${ }^{90} \mathrm{YCl}_{3}$; radiolabeled peptides such as ${ }^{90} \mathrm{Y}$-DOTA-RGDBBN; radiolabeled monoclonal antibodies such as ${ }^{89} \mathrm{Zr}$ DFO-J591; ${ }^{124}$ I-trastuzumab; and even a radionuclide-based imaging reporter gene-reporter probe system, herpes sim- 
plex virus type 1 thymidine kinase and ${ }^{18} \mathrm{~F}-\mathrm{FHBG}$, encompassing a wide range of radionuclides including $\beta^{+}$- and $\beta^{-}$-emitters.

\section{SPECT/CT}

Our group validated CLI with SPECT/CT by showing a good accumulation and retention of the clinically used agent $\mathrm{Na}^{131} \mathrm{I}$ in the thyroid of living mice with both OI and the fusion modality (12). Using the same radionuclide, $\mathrm{Hu}$ et al. performed a more thorough validation project of radioactive OI using SPECT along with an optical-CT multimodality system (16). They quantified CLI and SPECT signals by imaging radioactive sources consisting of glass vessels filled with a gradient amount of $\mathrm{Na}^{131} \mathrm{I}$, and the analysis showed an excellent correlation between the 2 modalities (radiance vs. counts $/ \mathrm{s}, R^{2}=0.98$ ). Hu et al. then proceeded to in vivo validation by implanting mice with aforementioned radioactive sources via laparotomy and then imaging and reconstructing the images using 3-dimensional Cerenkov luminescence tomography (CLT) based on a heterogeneous mouse model. They were able to show distance errors in the low millimeter range comparing CLT and SPECT reconstructions of locations of implanted radioactive sources.

\section{MRI}

Park et al. synthesized a novel triple-modality OI-PETMRI probe by linking a PET radionuclide, ${ }^{124} \mathrm{I}$, and a thermally cross-linked, superparamagnetic iron oxide nanoparticle for in vivo sentinel lymph node (SLN) imaging (17). In all 3 modalities, MRI metastatic SLNs presumably damaged or obliterated by tumor progression showed consistently lower signals than those of normal SLNs on the contralateral side of the animal.

\section{BLI}

As previously mentioned, our group incorporated BLI into validating radioactive OI along with PET (Supplemental Fig. 1A) (12). Our strategy was to use FLuc-transfected rat C6 glioma cells (C6-FLuc) that are capable of being imaged via both BLI and CLI. Although the 2 approaches use the same imaging modality, CLI and BLI can potentially be performed at the same time with little interference because radioactive OI signals can be detected in the wide spectral window of $\mathrm{CR}$.

\section{APPLICATIONS IN BIOMEDICAL RESEARCH}

\section{In Vivo Tumor Imaging}

Thus far, the single application of CLI that has enjoyed the most attention is unquestionably in vivo tumor imaging. The reasons for this phenomenon are severalfold. First, CLI, with its namesake, uses OI as its primary modality, which in itself is arguably the most widely available imaging modality for preclinical cancer research. Together with the fact that tumor imaging has been the most intensively studied subject in the field of molecular imaging, a marriage of CLI and tumor imaging should not be surprising. More- over, despite the fact that there have been only a few OI agents approved for clinical uses, there are many radioactive probes already widely in use in nuclear medicine; coincidentally, CLI elegantly uses luminescence generated from the decay of radionuclides and thus can take full advantage of the current armamentarium of PET/SPECT probes, most of which are directed at imaging molecular events relating to cancer. Because there are many publications that have used CLI for tumor imaging, it suffices to mention just a few here.

The proof-of-principle work by Robertson et al. is important because of their observation of CR from radionuclides not only in vitro but also in vivo, for which the authors performed CLI on a mouse bearing a CWR22-RV1 (human prostate tumor) xenograft after injecting ${ }^{18} \mathrm{~F}-\mathrm{FDG}$ (Supplemental Fig. 2A) (9). Our group was also able to accomplish in vivo tumor imaging using ${ }^{18} \mathrm{~F}-\mathrm{FDG}$ as the radiotracer (Supplemental Figs. 1A-1C; "Recent Advances in CLI" section) (12). Additionally, Park et al. demonstrated specific uptake of ${ }^{124} \mathrm{I}$-labeled trastuzumab by a HER2expressing tumor (Supplemental Fig. 2B) (15). As previously mentioned, Ruggiero et al. developed the ${ }^{89} \mathrm{Zr}$-labeled monoclonal antibody ${ }^{89} \mathrm{Zr}$-DFO-J591 and demonstrated specific tumor uptake using the inherent CR emitted from this novel radiolabeled immunoconjugate (Supplemental Figs. 2C and 2D; "Cross Validations of CLI" section) (14). As another illustration of the opportunities that CLI makes possible in tumor imaging, Boschi et al. studied an experimental mammary cancer model, BB1, and performed dynamic ${ }^{18} \mathrm{~F}$-FDG uptake measurements in mice carrying these tumors (Supplemental Fig. 2E) (18). The authors plotted time-activity curves of tumor versus non-tumor-bearing contralateral sides as well as several internal organs including the brain and kidneys and showed that the time-activity curves matched well with the 2-compartment irreversible trapping model of ${ }^{18} \mathrm{~F}-\mathrm{FDG}$. They then suggested that dynamic measurements via CLI could provide valuable information of the pharmacokinetics of the injected biomarker.

\section{Using CR as Excitation Source}

Although CR can be directly registered and quantified by OI, the possibility of using CR as a primary source of light, that is, using CR to excite a secondary light-emitting agent, has always been intriguing. Quantum dots (QDs) are great candidates for this application of CLI because of both their advantages and their limitations. First, QDs as an OI agent display many favorable characteristics such as sizedependent tunable emission bands, high photo stability, high-fluorescence quantum yields, wide excitation spectra, a large molar-extinction coefficient, and large Stokes shifts. Consequently, QDs have been explored in the context of a variety of biomedical applications including detection of biomolecules, tissue staining, and molecular imaging $(19,20)$. However, much like other fluorescent probes, external light is required to illuminate QDs, limiting the in vivo applicability of QDs because of significant autofluorescence, poor tissue penetration, scattering, and absorption 


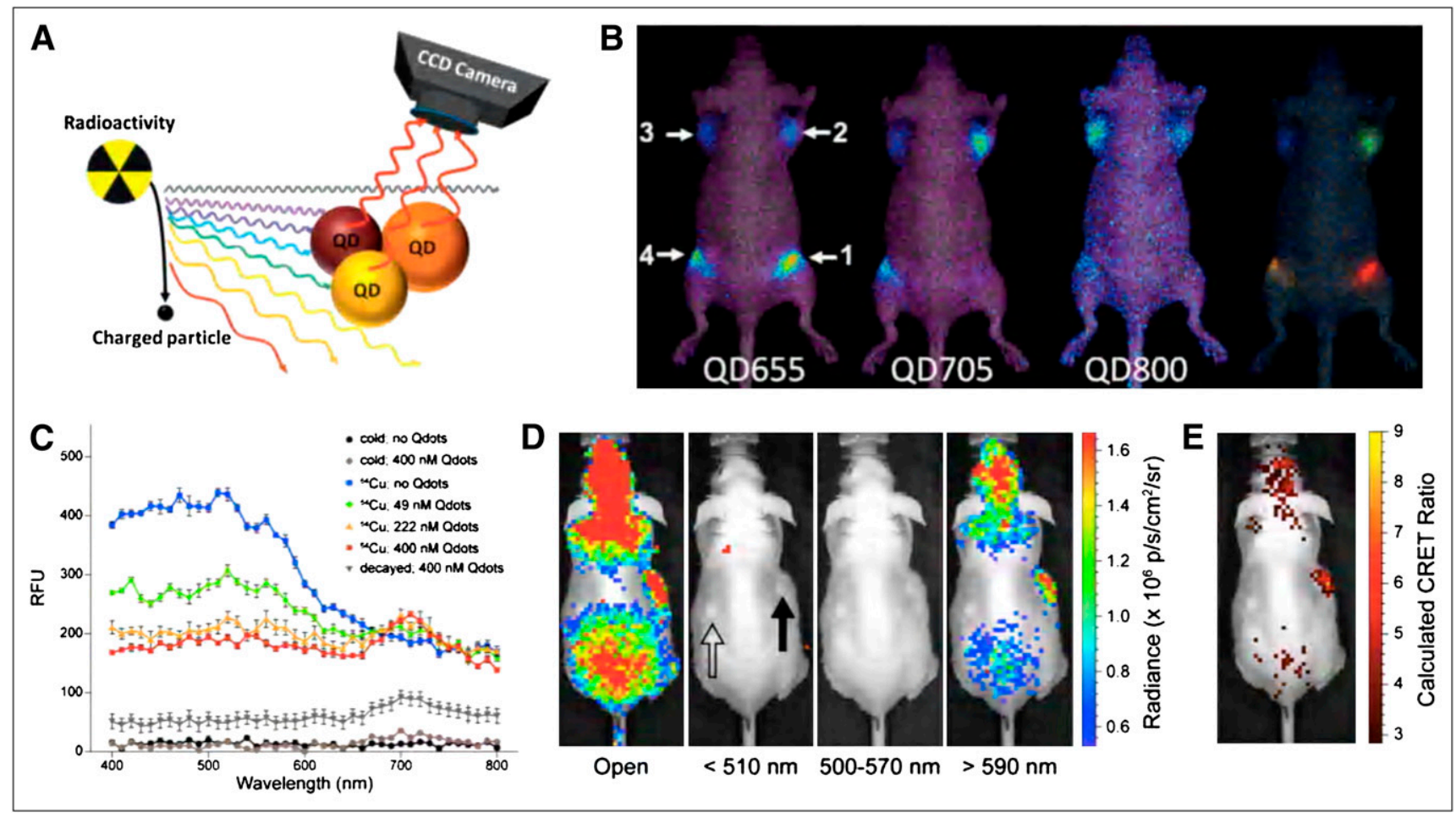

FIGURE 3. CR as excitation source. (A) Illustration of radiation luminescence-excited QDs for OI. Four samples were injected intramuscularly to different locations of mice. (B) Multiplexed in vivo radioactivity illuminated QD imaging. Four samples were injected intramuscularly to different locations of mice (1, QD655/Na131l; 2, QD705/Na131I; 3, QD800/Na131I; and 4, QD655/QD705/QD800/Na131I). For all samples, radioactivity is $0.37 \mathrm{MBq}$. From left to right, in vivo radioactivity illuminating spectral imaging of QD655, QD705, QD800, and spectral unmixed image of same mouse. (C) Ultraviolet/visible emission spectra of ${ }^{64} \mathrm{CuCl}_{2}$ in PBS containing various concentrations of Qtracker705 nanoparticles (Qdots) demonstrate CRET. Blue $={ }^{64} \mathrm{CuCl}_{2}$ without Qdots; green $={ }^{64} \mathrm{CuCl}_{2}$ with $49 \mathrm{nM}$ Qdots; orange $={ }^{64} \mathrm{CuCl}_{2}$ with $222 \mathrm{nM}$ Qdots; red $={ }^{64} \mathrm{CuCl}_{2}$ with $400 \mathrm{nM}$ Qdots; black = nonradioactive $\mathrm{CuCl}_{2}$ without Qdots; brown = nonradioactive $\mathrm{CuCl}_{2}$ with $400 \mathrm{nM}$ Qdots; gray = decayed ${ }^{64} \mathrm{CuCl}_{2}$ with $400 \mathrm{nM}$ Qdots. (D) Subcutaneous pseudotumors of $500 \mathrm{nM}$ Qtracker705-impregnated Matrigel (BD Biosciences) (closed arrow) and PBS-impregnated Matrigel (open arrow) in opposing flanks of nu/nu mice were imaged with IVIS 100 using open ( $<510 \mathrm{~nm}$ [blue], $500-570 \mathrm{~nm}$ [green], and $>590 \mathrm{~nm}$ [red]) filters $30 \mathrm{~min}$ after tail-vein injection of ${ }^{18} \mathrm{~F}-\mathrm{FDG}(17.6 \mathrm{MBq} ; 475 \mu \mathrm{Ci})$. (E) Calculated CRET image. (A and B adapted with permission of (23) and C-E adapted with permission of (24).) CCD = charge-coupled device.

of excitation photons. Thus, self-illuminating QD conjugates, compared with regular QDs, will be superior for in vivo studies, and, indeed, several such QD conjugates have already been described by mimicking bioluminescence resonance energy transfer, during which chemical energy is converted into photons that in turn excite the QDs $(21,22)$. Our group thus hypothesized that CR from radionuclides such as the $\beta^{-}$-emitter ${ }^{131} \mathrm{I}$, especially signals in the visible and near infrared (NIR) ranges (although CR is dominated by emission in ultraviolet and blue regions, its spectrum does extend to NIR ranges), could be used to illuminate fluorescent materials such as QDs, with corresponding absorption spectra for applications both in vitro and in vivo (Fig. 3A) (23).

CR from $\mathrm{Na}^{131}$ I was first demonstrated in vitro to excite QDs (QD655, QD705, and QD800, with QD655 being the most sensitive of the 3) in both phosphate-buffered saline (PBS) and mouse serum. This secondary emission from QDs was then found to be detectable by OI in vivo, with a subcutaneous injection of the $\mathrm{QD} / \mathrm{Na}^{131} \mathrm{I}$ concoction having higher sensitivity than the intramuscular injection.
Multiplex imaging was also tested using all 3 QDs along with $\mathrm{Na}^{131} \mathrm{I}$ both in vitro and in vivo, and the results were promising (in vivo results shown in Fig. 3B).

Dothager et al. also explored the potential of using CR as an excitation source (24). They hypothesized that high Stokes-shift QDs can be spectrally coupled to CR and essentially red-shift the emission spectrum of CR and make CLI more sensitive in vivo. Borrowing the concept of bioluminescence resonance energy transfer, they went on further to define CR energy transfer (CRET) as the normalized ratio of optical signals detected within a spectral region centered on the emission of fluorophore over optical signals detected within a spectral region centered on the emission of CR using the following formula:

$$
\text { CRET }_{x}=\frac{\text { Cerenkov }+ \text { Fluorophore }_{X}}{\text { Cerenkov }+ \text { Fluorophore }_{Y}}-\frac{\text { Cerenkov }_{X}}{\text { Cerenkov }_{Y}} . \quad \text { Eq. } 3
$$

Here $\mathrm{X}$ stands for the spectral window centered on fluorophore emission, and $\mathrm{Y}$ stands for the spectral window centered on CR emission. 
They then demonstrated that the addition of QDs (Qtracker705) to ${ }^{64} \mathrm{Cu}-\mathrm{CuCl}_{2}$ in $\mathrm{PBS}$ resulted in a predicted shift of the emission spectrum of $\mathrm{CR}$ from ${ }^{64} \mathrm{Cu}$, with the formation of a new peak at $705 \mathrm{~nm}$ whose height was also dependent on the concentration of QDs (Fig. 3C). Using ${ }^{18} \mathrm{~F}-\mathrm{FDG}$, the authors then showed that photon flux and CRET ratio (calculated using Eq. 3) correlated positively with both concentrations of QDs and the radioisotope. Using subcutaneous pseudotumor phantoms impregnated with different concentrations of QDs along with PBS alone, Dothager et al. demonstrated in vivo that CRET allowed CLI signals from ${ }^{18} \mathrm{~F}$-FDG to be enhanced in the red region of the visible spectrum (Figs. 3D and 3E). Considering that the mean distance traveled by positrons emitted by ${ }^{18} \mathrm{~F}$ and ${ }^{64} \mathrm{Cu}$ is around $0.9 \mathrm{~mm},{ }^{18} \mathrm{~F}$-FDG does not need to be proximate to the QDs for a CRET signal to be generated, which is certainly an added advantage of this approach. However, there are still unknowns surrounding this interesting phenomenon. For instance, the relationship between the concentrations of QDs and CRET ratios in vitro was measured to be linear but linearity was somehow lost in vivo. The authors suggested that tissue absorption and scattering could contribute to this inconsistency. Moreover, although resonance could be used to explain the CRET phenomenon, the exact mechanisms still demand further investigations because some other high-energy radiation sources, such as Bremsstrahlung and $\gamma$-radioluminescence, could be playing a role as well.

\section{OI of Reporter Gene Expression}

As a well-established method for noninvasive imaging of gene expression, reporter gene-reporter probe technology has numerous applications in drug screening, disease monitoring, cell tracking, and more $(2,25)$. For instance, the gene-reporter probe system luciferase-D-luciferin has enjoyed much popularity using BLI, featuring both high sensitivity and low background (25). Yet the enzymesubstrate reaction usually produces light, with peaks some distance away from the red and NIR regions, and thus suffers from significant tissue absorption and scattering when the technique is applied in living animals (26). In nuclear imaging, 2 of the most widely used reporter gene-reporter probe pairs are ${ }^{18} \mathrm{~F}$-FHBG and $2{ }^{\prime}{ }^{18} \mathrm{~F}$-fluoro-5-ethyl-1- $\beta$-Darabinofuranosyluracil $(27,28)$. Although these systems circumvent the shortcomings of their counterparts in BLI, they too have disadvantages in that the nuclear imaging instruments such as PET and SPECT impose high costs to researchers and thus are often not accessible. As briefly mentioned in the "Cross Validations of CLI" section, our group thus studied the possibility of using CLI as a means of combining the advantages of optical and nuclear reporter gene expression technologies (13). The C6 rat glioma cell line stably transfected with HSV-sr39tk plasmids (C6-tk) was used (29). ${ }^{18} \mathrm{~F}$-FHBG uptake was measured by both OI and $\gamma$-counter in vitro, and PET and OI were used for in vivo imaging studies. Consistent with the good in vitro detection capability of OI of the radioactive reporter genereporter probe system, mice bearing C6-tk can be imaged by both PET (T/N, $43.2 \pm 8.4$ at $1 \mathrm{~h}$ and $143.0 \pm 14.8$ at $2 \mathrm{~h}$ ) and $\mathrm{OI}(\mathrm{T} / \mathrm{N}, 2.6 \pm 0.5$ at $1 \mathrm{~h}$ and $5.1 \pm 1.1$ at $2 \mathrm{~h})$ after tail vein injection of ${ }^{18} \mathrm{~F}$-FHBG (Supplemental Fig. 3).

Our report demonstrates the feasibility of using CLI to monitor a radiolabeled reporter gene-reporter probe system, this novel approach can potentially provide 3-dimensional OI reconstruction superior to the traditional BLI probe systems thanks to the characteristic extension of the CR spectrum into tissue-penetrating red and NIR regions.

\section{Radioactive Source-Depth Measurement In Vivo and In Vitro}

In addition to derivation of a detailed model of the CR spectrum by considering positron energy distribution, Spinelli et al. also presented 2 methods of source-depth measurement of CLI-one based on the wavelengthdependent tissue scattering and the other based on the characteristics of the CR spectrum (10). Using the standard diffusion approximation of light propagation in a slab sample while approximating high-frequency weighted tissue scattering as the predominant phenomenon, Spinelli et al. derived the first method of measurement. Then they proposed the second approach using total integrated radiance from source and surface at 2 different wavelengths and combining the data with the known CR spectral distribution of $1 / \lambda^{2}$. The first method yielded a measurement of $1 \mathrm{~mm}$ for the depth of the heart of a mouse, with a relative uncertainty of 0.25 , and the second method gave a measurement of $1.6 \mathrm{~mm}$; the gold standard measurement using MRI yields $1.5 \mathrm{~mm}$. The authors also pointed out that accuracy of the measurements was better than expected because a mouse is quite different from the assumed homogeneous flat slab model.

Although Spinelli et al. measured the depth of heart as a proof of principle of their proposed approaches of depth measurement, Boschi et al. attempted to broaden the application of CLI depth measurement by measuring the depth of tumor in living mice using these 2 methods (18). Although a quasi in vivo measurement of depth of ${ }^{18} \mathrm{~F}$ FDG-filled capillaries in chicken breasts yielded good results using both methods, only the second method gave a reasonable estimation of the depths of tumor and brain in living mice.

Overall, the explorations into CLI depth measurement by these 2 groups are promising, but the simplification of the heterogeneous living tissue into a homogeneous slab sample may carry some caveats. Thus, development of more accurate alternative methods is certainly called for if CLI depth measurement is to be used in intraoperative applications such as endoscopies.

\section{CLT}

In addition to source-depth approximation using straight mathematic derivations such as multispectral analysis, another potential approach to obtain spatial information 
of the source of CR is via tomography, similar to the known technologies such as bioluminescent optical tomography and fluorescent optical tomography. Li et al. first explored this possibility using an inverse algorithm to reconstruct the distribution of CLI tracers from surface measurements, and they named this novel approach CLT $(30,31)$. Using the diffusion equation that has been derived for fluorescent optical tomography (31), they also made an assumption that the inside of a mouse was homogeneous and uniform. Then, as a proof of principle they constructed a phantom mimicking tissue scattering and absorption and used CLI surface measurements of implanted ${ }^{18} \mathrm{~F}$-FDG to reconstruct source distribution. By applying Tikhonov regularization, they were able to solve the ill-posed inverse problem iteratively with a preconditioned conjugate gradient method (31). By comparing with the PET image of the same phantom, CLT reconstructed the source of activity in the actual location, albeit with lower spatial resolution than that of PET. Then Li et al. applied CLT in mice with a human melanoma DX-3 xenograft on the left dorsal flank and were also able to reconstruct the source of CR near the tumor but again with some artifacts (Supplemental Figs. 4A and 4B). They attributed the inaccuracies to the lead bricks they placed between the source and the charge-coupled device camera for blocking $511-\mathrm{keV}$ $\gamma$-rays, a lack of spectral information, and the assumption of homogeneity in the photon propagation model of CLT.

The last concern of $\mathrm{Li}$ et al. about their inverse algorithm was soon addressed by $\mathrm{Hu}$ et al. (16). Their use of SPECT to validate 3 -dimensional reconstruction results was briefly mentioned in the "Cross Validations of CLI" section. More important, these authors reconstructed a 3-dimensional model that included major internal organs from CT images and mapped the acquired Cerenkov luminescence images onto the heterogeneous model and thus avoided the assumption of homogeneity (Supplemental Figs. 4C-4E). After selecting an initial permissible source region $\Omega$ from surface measurements, Hu et al. used a diffusion equationbased, adaptive $h p$-finite element method to recover the 3dimensional distribution of the implanted $\mathrm{Na}^{131}$ I source in living mice and were able to achieve a small distance error when compared with the actual location imaged by CT $(2.15 \mathrm{~mm})$. Notably, the authors applied the same algorithm using a homogeneous model and observed a significantly larger error $(4.41 \mathrm{~mm})$.

Thanks to the work of $\mathrm{Li}$ et al. and $\mathrm{Hu}$ et al., CLT has been developed as a novel CLI technology, with both complementarities with existing OI tomography technologies and virtues of its own. Traditional OI tomography such as bioluminescent optical tomography is largely applied in reporter gene imaging because of its own characteristics and limitations whereas CLT can be useful for monitoring radiopharmaceuticals, especially in preclinical in vivo applications. Furthermore, CLT naturally uses radiotracers, many of which have been tested and used in clinics for years, and thus avoids potential toxicities that have long plagued several traditional bioluminescent and fluorescent probes. Yet CLT is far from a mature technology-it still suffers from relatively low spatial resolution due to tissue scattering and absorption and mathematic compromises that have to be made to solve the ill-posed inverse problem for tomographic reconstruction.

\section{High-Resolution Imaging}

The effort by Cho et al. on using CR to measure $\beta$-particles in a microfluidic chip was briefly mentioned in the "Recent Advances in CLI" section. A microfluidic chip is an example of a lab-on-a-chip device that integrates laboratory functions, such as analyzing molecular processes and synthesizing biomolecules, while dealing with nanoliter levels of fluid. One particular application of microfluidic chips is on-demand synthesis of ${ }^{18} \mathrm{~F}$-labeled biomolecules for PET (32). However, the development of this technology has been limited by the lack of an effective imaging system to monitor loss of radioactivity, trapped fluid, and imperfections of chips. The authors cleverly hypothesized that CR could be detected from ${ }^{18} \mathrm{~F}$ with high sensitivity, and indeed they found resolution of CLI of microfluidic chips is good and dominated by the physical size of the channel $(200 \mu \mathrm{m})$, because the measured full width at half maximum was $349.4 \mu \mathrm{m}$ (Figs. 1C and 1D). Signal calibration curve, minimum detectable activity, and dynamic range were also measured to evaluate $\mathrm{CR}$ as an imaging tool of microfluidic chips. The authors pointed out that low signal intensity of CR can be an issue in other applications, but in radiochemical synthesis the required activity concentrations are most likely well above the measured minimum detectable activity and thus CR can be useful in monitoring the progress of PET probe synthesis in microfluidic chips. Although this work focused on microfluidic chips, there are many potential applications of CR in high-resolution imaging of cells, tissues, and other small devices.

\section{PERSPECTIVES}

\section{Advantages of CLI}

As a technique that uses OI, CLI enjoys many of the advantages that belong to this imaging modality: high sensitivity, ease of learning and use, low cost and wide availability, short acquisition time, and relatively high throughput. These qualities of OI can render CLI a powerful tool to screen a large number of drugs and probes, particularly in terms of their biodistribution and pharmacokinetics. It is reasonable to propose a general screening scheme for which only the drugs or probes found during the screening process by CLI to satisfy certain criteria would be granted detailed investigations by such modalities as PET and MRI. It is foreseeable that by minimizing the use of the modalities that are more expensive, are less available, and have lower throughput, the screening process of drugs and probes will be significantly accelerated. There will be no shortage of potential probes approved by the Food and Drug Administration for CLI, because CLI uses nuclear 
probes and several PET and SPECT probes have long been approved. Because of the intrinsic ability of CLI to image radioactivity, many drugs and biologic probes labeled by radionuclides can now be monitored by OI, and these include some of the most widely used nuclides in nuclear medicine (e.g., ${ }^{18} \mathrm{~F},{ }^{131} \mathrm{I}$, and ${ }^{90} \mathrm{Y}$ ). Notably, pure $\beta$-emitters such as ${ }^{90} \mathrm{Y}$ and ${ }^{32} \mathrm{P}$, which have been widely studied in nuclear medicine yet have traditionally been difficult to image, can now be easily detected thanks to CLI, without the hassles of invasive methods such as liquid scintillation and Cerenkov counting (Supplemental Figs. 1D and 1E).

Compared with traditional OI, radioactive OI has several advantages of its own. For spectral distribution, CR is most intense in the ultraviolet or blue region but does have significant extension into the green or red region and thus makes radioactive OI relatively more feasible in living animals because light in that part of the spectrum has the best penetration. Also, thanks to the characteristic continuous spectrum, CLI possesses a wide window of observation regarding wavelength when compared with conventional fluorescence imaging and BLI. Because of this wide window, researchers can perform CLI and other conventional OI techniques such as BLI or fluorescent imaging simultaneously when desired. Moreover, toxicities of traditional fluorescent and bioluminescent probes remain to be studied and can potentially complicate clinical applications; on the other hand, several radiotracers suitable for CLI have long been approved and used in clinics. Notably, fluorescent dyes often possess bulky structures, which can potentially alter functions of the biomolecule of interest; with radioactive OI, one can simply label the molecule with a radioisotope, effectively avoiding bulky conjugates while achieving dual-modality capability for imaging. An intrinsic quality of CLI is that the radioactive probes are essentially self-luminescent, and thus no external excitation light is needed. The advantages stemming from this quality are 2-fold: the self-luminescence can be used as a primary source of excitation light that in turn excites a secondary source of light such as QD, which in and of itself can potentially improve the sensitivity of CLI $(23,24)$, and the freedom of excitation light can significantly reduce the extent of autofluorescence as well as the complexity of experimental design. Last, considering the physics of CR in the case of positron emitters, because signals from CLI are produced earlier than the annihilation event, CLI should yield more accurate localization and potentially higher spatial resolution than PET. The application of CLI in microfluidic chips by Cho et al. is a good example; however, one can easily think of many other potential applications for which an accurate localization is needed such as high-resolution tissue imaging and imaging of other microscale devices.

\section{Disadvantages of CLI}

Because CLI enjoys the intrinsic advantages of OI, this technique also naturally suffers from many of the disadvan- tages of its designated modality. Although the continuous spectrum of CR does extend into the green and red regions, signal intensity in that part of the spectrum is limited by the $1 / \lambda^{2}$ relationship. In contrast, the $511-\mathrm{keV}$ photons tracked by PET have minimal tissue absorption and scattering, and thus in this respect the sensitivity of CLI is significantly lower. In addition, according to calculations by Robertson et al., the sensitivity of radioactive OI is merely $10 \%$ of that of PET (9). A consequence of this limitation is that CLI will suffer from poorer quantification than other nuclear imaging modalities. However, preclinical research, such as studies involving subcutaneous xenografts in small animals as well as clinical studies and applications regarding superficial diseases, could still be accomplished with high sensitivity and good quantification capability using radioactive OI. Additionally, CR can stimulate local autofluorescence in living tissues, which can be disadvantageous in many aspects including, but not limited to, spatial resolution and signal specificity. This phenomenon could also be used to researchers' advantage as a primary source of excitation light. Another notable characteristic of in vivo radioactive OI is the significantly reduced signals from high-blood-content organs such as the liver, kidneys, and spleen. This reduction happens because the high concentration of hemoglobin in these organs tends to absorb most of the luminescent signal. This phenomenon has mostly been considered to be one of the disadvantages of radioactive OI, especially when the disease processes of interest evolve in the realm of these organs. However, with the advent of CLI this phenomenon can potentially become an advantage, particularly in preclinical studies in which the position of the area of interest is predetermined and can be intentionally placed away from the aforementioned internal organs. In this respect then, with the help of PET, which is excellent in visualizing internal organs including the liver and kidneys, CLI can complement PET by visualizing the area of interest such as a tumor without signal interferences from nearby organs. Last, just as CLI suffers from some of the shortcomings of traditional OI because of its dependence on OI as its sole modality, CLI also suffers from the intrinsic disadvantages of radiotracers that are essential to the technique. These disadvantages include, but are not limited to, the dangers of radioactive contamination in research settings and radiation poisoning in clinical settings.

\section{Future Applications of CLI}

Perhaps the most promising applications of CLI lie in drug and tracer development and screening. Many clinically approved radionuclides have been available for both diagnosis and treatment of cancer, and now with radioactive OI, the development and screening of new radiotracers can be speeded up. CLI can be effective not only for screening drugs and tracers based on their biodistribution and pharmacokinetics but also for monitoring the therapeutic effects of candidate drugs. Our group has recently found that in both treated and untreated mice with xenografts 
(treatment was performed with a well-established antineoplastic regimen), there exists an excellent correlation between CLI luminescent signals and PET quantifications of the tumors as well as $\mathrm{T} / \mathrm{N}$ organ ratios (33). CLI will allow researchers to easily label the biologic compounds of interest, significantly reduce cost of instrumentation, notably shorten scan time, and improve throughput. In high-blood-content organs in which CLI is disadvantaged, PET can complement with both imaging and quantification.

These currently approved radionuclides can also benefit from CLI in an important way. Currently, for intraoperative monitoring of radionuclides surgeons can use only handheld devices that unfortunately do not provide any spatial information. Thus, it has long been implicated that realtime guidance during resection can significantly benefit surgical applications such as endoscopies and endoscopic surgeries. However, there are no clinically approved probes for this application despite the presence of fluorescence molecular tomography and optical fiber-based technologies. Because of the emergence of CLI, the development of intraoperative OI now has one fewer significant obstacleno OI agents need to be specifically approved because of the availability of approved radionuclides - and thus it will be exciting to see CLI providing surgeons with vital information about tumors, metastasis, and radiopharmaceuticals during surgeries in the near future.

\section{DISCLOSURE STATEMENT}

The costs of publication of this article were defrayed in part by the payment of page charges. Therefore, and solely to indicate this fact, this article is hereby marked "advertisement" in accordance with 18 USC section 1734.

\section{ACKNOWLEDGMENTS}

We thank Dr. Sanjiv Sam Gambhir for his helpful discussion. This study was supported in part by the National Cancer Institute (NCI) (R21 CA121842) and a Stanford Medical Scholar Research Fellowship. No other potential conflict of interest relevant to this article was reported.

\section{REFERENCES}

1. Weissleder R, Pittet MJ. Imaging in the era of molecular oncology. Nature. 2008;452:580-589.

2. Pysz MA, Gambhir SS, Willmann JK. Molecular imaging: current status and emerging strategies. Clin Radiol. 2010;65:500-516.

3. Lucignani G. Cerenkov radioactive optical imaging: a promising new strategy. Eur J Nucl Med Mol Imaging. 2011;38:592-595.

4. Cerenkov PA. Visible radiation produced by electrons moving in a medium with velocities exceeding that of light. Phys Rev. 1937;52:378-379.

5. Cerenkov PA. Visible emission of clean liquids by action of $\gamma$-radiation. Dokl Akad Nauk SSSR 2. 1934;2:451-454.

6. Elrick RH, Parker RP. The use of Cerenkov radiation in the measurement of betaemitting radionuclides. Int J Appl Radiat Isot. 1968;19:263-271.
7. Jelley JV. Cerenkov radiation and its applications. Br J Appl Phys. 1955;6:227232.

8. Ross HH. Measurement of beta-emitting nuclides using Cerenkov radiation. Anal Chem. 1969;41:1260-1265.

9. Robertson R, Germanos MS, Li C, Mitchell GS, Cherry SR, Silva MD. Optical imaging of Cerenkov light generation from positron-emitting radiotracers. Phys Med Biol. 2009;54:N355-N365.

10. Spinelli AE, D'Ambrosio D, Calderan L, Marengo M, Sbarbati A, Boschi F. Cerenkov radiation allows in vivo optical imaging of positron emitting radiotracers. Phys Med Biol. 2010;55:483-495.

11. Cho JS, Taschereau R, Olma S, et al. Cerenkov radiation imaging as a method for quantitative measurements of beta particles in a microfluidic chip. Phys Med Biol. 2009;54:6757-6771.

12. Liu H, Ren G, Miao Z, et al. Molecular optical imaging with radioactive probes. PLOS ONE. 2010;5:e9470.

13. Liu H, Ren G, Liu S, et al. Optical imaging of reporter gene expression using a positron-emission-tomography probe. J Biomed Opt. 2010;15:060505.

14. Ruggiero A, Holland JP, Lewis JS, Grimm J. Cerenkov luminescence imaging of medical isotopes. J Nucl Med. 2010;51:1123-1130.

15. Park JC, Il An G, Park S-I, et al. Luminescence imaging using radionuclides: a potential application in molecular imaging. Nucl Med Biol., in press.

16. Hu Z, Liang J, Yang W, et al. Experimental Cerenkov luminescence tomography of the mouse model with SPECT imaging validation. Opt Express. 2010;18: 24441-24450.

17. Park JC, Yu MK, An GI, et al. Facile preparation of a hybrid nanoprobe for triple-modality optical/PET/MR imaging. Small. 2010;6:2863-2868.

18. Boschi F, Calderan L, D'Ambrosio D, et al. In vivo ${ }^{18} \mathrm{~F}-\mathrm{FDG}$ tumour uptake measurements in small animals using Cerenkov radiation. Eur J Nucl Med Mol Imaging. 2011;38:120-127.

19. Michalet X, Pinaud FF, Bentolila LA, et al. Quantum dots for live cells, in vivo imaging, and diagnostics. Science. 2005;307:538-544.

20. Medintz IL, Uyeda HT, Goldman ER, Mattoussi H. Quantum dot bioconjugates for imaging, labelling and sensing. Nat Mater. 2005;4:435-446.

21. So MK, Xu C, Loening AM, Gambhir SS, Rao J. Self-illuminating quantum dot conjugates for in vivo imaging. Nat Biotechnol. 2006;24:339-343.

22. Xing Y, Rao J. Quantum dot bioconjugates for in vitro diagnostics \& in vivo imaging. Cancer Biomark. 2008;4:307-319.

23. Liu H, Zhang X, Xing B, Han P, Gambhir SS, Cheng Z. Radiation-luminescenceexcited quantum dots for in vivo multiplexed optical imaging. Small. 2010;6: 1087-1091.

24. Dothager RS, Goiffon RJ, Jackson E, Harpstrite S, Piwnica-Worms D. Cerenkov radiation energy transfer (CRET) imaging: a novel method for optical imaging of PET isotopes in biological systems. PLoS ONE. 2010;5:e13300.

25. Min JJ, Gambhir SS. Molecular imaging of PET reporter gene expression. Handb Exp Pharmacol. 2008;(185 pt 2):277-303.

26. Hastings JW. Chemistries and colors of bioluminescent reactions: a review. Gene. 1996;173(1 spec no):5-11.

27. Gambhir SS, Barrio JR, Phelps ME, et al. Imaging adenoviral-directed reporter gene expression in living animals with positron emission tomography. Proc Natl Acad Sci USA. 1999;96:2333-2338.

28. Tjuvajev JG, Finn R, Watanabe K, et al. Noninvasive imaging of herpes virus thymidine kinase gene transfer and expression: a potential method for monitoring clinical gene therapy. Cancer Res. 1996;56:4087-4095.

29. Gambhir SS, Bauer E, Black ME, et al. A mutant herpes simplex virus type 1 thymidine kinase reporter gene shows improved sensitivity for imaging reporter gene expression with positron emission tomography. Proc Natl Acad Sci USA. 2000;97:2785-2790.

30. Li C, Mitchell GS, Cherry SR. Cerenkov luminescence tomography for smallanimal imaging. Opt Lett. 2010;35:1109-1111.

31. Li C, Mitchell GS, Dutta J, Ahn S, Leahy RM, Cherry SR. A three-dimensional multispectral fluorescence optical tomography imaging system for small animals based on a conical mirror design. Opt Express. 2009;17:7571-7585.

32. Whitesides GM. The origins and the future of microfluidics. Nature. 2006; 442:368-373.

33. Xu Y, Chang E, Liu H, Jiang H, Gambhir SS, Cheng Z. Proof-of-concept study of monitoring cancer drug therapy with Cerenkov luminescence imaging. $\mathrm{J} \mathrm{Nucl}$ Med. In press. 